\title{
Spectral Integration in the Inferior Colliculus: Role of Glycinergic Inhibition in Response Facilitation
}

\author{
Jeffrey J. Wenstrup and Scott A. Leroy \\ Department of Neurobiology and Pharmacology, Northeastern Ohio Universities College of Medicine, Rootstown, Ohio \\ 44272
}

This study examined the contribution of glycinergic inhibition to the time-sensitive spectral integration performed by neurons in the inferior colliculus of the mustached bat (Pteronotus parnellii). These neurons are sometimes called combination-sensitive because they display facilitatory (or inhibitory) responses to the combination of distinct spectral elements in sonar or social vocalizations. Present in a wide range of vertebrates, their temporally and spectrally selective integration is thought to endow them with the ability to discriminate among social vocalizations or to analyze particular cues concerning sonar targets. The mechanisms that underlie these responses or the sites in the auditory system where they are created are not known.

We examined combination-sensitive neurons that are facilitated by the presentation of two different harmonic elements of the bat's sonar call and echo. Responses of 24 single units were recorded before and during local application of strych-

Communication by sound uses spectrally and temporally complex signals, and their analyses in the CNS require integration across the spectral and temporal elements in the signals. One form of integration is performed by neurons responding best when distinct spectral elements in signals are presented in specific temporal relationships. These neurons are sometimes called combinationsensitive. Although general features of combination-sensitive responses are common in a wide range of vertebrate species, the species-specific features of the integration create response preferences to a bird's own song (Margoliash and Fortune, 1992; Doupe, 1997), to pulse-echo delay in mustached bats (O'Neill and Suga, 1982; Olsen and Suga, 1991), and among different social vocalizations in frogs (Fuzessery and Feng, 1983) and mammals (Rauschecker et al., 1995; Ohlemiller et al., 1996). The neural interactions that underlie these response properties are poorly understood, but have been generally thought to originate in the auditory forebrain (Olsen, 1992; Winer et al., 1995; Rauschecker, 1998).

\footnotetext{
Received July 7, 2000; revised Oct. 25, 2000; accepted Nov. 13, 2000.

This work was supported by National Institutes of Health Grant 5 R01 DC 00937 from the National Institute on Deafness and Other Communication Disorders. We thank Z. M. Fuzessery and C. V. Portfors for helpful comments on this manuscript, J. C. Hall for instruction on the microiontophoretic technique, C. D. Grose for technical assistance, F.-M. Chen for software, and Ministry of Agriculture, Land, and Marine Resources of Trinidad and Tobago for permission to export the bats.

Correspondence should be addressed to Jeffrey J. Wenstrup, Department of Neurobiology and Pharmacology, Northeastern Ohio Universities College of Medicine, 4209 State Route 44, Rootstown, Ohio 44272-0095. E-mail: jjw@neoucom.edu.

Dr. Leroy's present address: Abbott Laboratories, Department 9L9, Building AP32-LL, 200 Abbott Park Road, Abbott Park, IL 60064.

Copyright (C) 2001 Society for Neuroscience $0270-6474 / 01 / 210001-\bullet \$ 15.00 / 0$
}

nine, an antagonist of glycinergic inhibition. For each of the 24 units, strychnine application eliminated or greatly reduced temporally sensitive facilitation. There was no difference in this effect for neurons tuned to frequencies associated with the frequencymodulated or the constant-frequency sonar components.

These results are unusual because glycine is considered to be an inhibitory neurotransmitter, but here it appears to be essential for the expression of combination-sensitive facilitation. The findings provide strong evidence that facilitatory combination-sensitive response properties present throughout the mustached bat's auditory midbrain, thalamus, and cortex originate through neural interactions in the inferior colliculus.

Key words: auditory pathways; bat; combination-sensitive; complex sounds; frequency integration; glycine; inferior colliculus; mustached bat; spectral integration; strychnine

This study examines mechanisms that contribute to combinationsensitive response properties in the mustached bat (Pteronotus parnellii). In this species, combination-sensitive responses are thought to participate in analyses of both sonar (O'Neill and Suga, 1982; Suga et al., 1983) and social (Ohlemiller et al., 1996; Esser et al., 1997) vocalizations. In the context of sonar, most combination-sensitive neurons display facilitated responses when a lower frequency signal in the range of the first sonar harmonic $(24-31 \mathrm{kHz})$ is presented at a specific time relative to a higher frequency signal in ranges associated with higher harmonics of sonar echoes. This response property may extract information about target features such as distance and movement (O'Neill and Suga, 1982; Suga et al., 1983). As well as occurring in the auditory cortex and thalamus, combination-sensitive neurons are abundant in the mustached bat's inferior colliculus (IC) (Mittmann and Wenstrup, 1995; Yan and Suga, 1996; Portfors and Wenstrup, 1999a). Their abundance and similarity to cortical and thalamic combination-sensitive neurons in this species suggest that the IC,

This article is published in The Journal of Neuroscience, Rapid Communications Section, which publishes brief, peer-reviewed papers online, not in print. Rapid Communications are posted online approximately one month earlier than they would appear if printed. They are listed in the Table of Contents of the next open issue of JNeurosci. Cite this article as: JNeurosci, 2001, 21: RC124 (1-6). The publication date is the date of posting online at www.jneurosci.org.

http://www.jneurosci.org/cgi/content/full/4941 
or the auditory brainstem below it, is the locus of integrative mechanisms that create temporally sensitive facilitation between spectrally distinct inputs.

To investigate these integrative mechanisms, we recorded combination-sensitive responses of single units in the IC before and after local application of strychnine, an antagonist of glycinergic inhibition. We studied IC neurons because anatomical evidence from retrograde transport studies (Wenstrup et al., 1999) and physiological recordings of lateral lemniscal nuclei (Portfors and Wenstrup, 1999b) suggest that these responses originate in the IC. We examined the role of inhibitory mechanisms because physiological evidence suggested that inhibition may play a role in combination-sensitive facilitation. For example, facilitation by the lower frequency signal was sometimes preceded by strong inhibition (Olsen and Suga 1991; Portfors and Wenstrup, 1999a). Other combination-sensitive neurons show only inhibitory influences of the lower frequency signals (O’Neill, 1985; Mittmann and Wenstrup, 1995; Portfors and Wenstrup, 1999a). The role of glycinergic inhibition was examined because it could implicate brainstem as opposed to midbrain or cortical inputs to these IC neurons; glycinergic immunopositive cell bodies are not present in midbrain or forebrain auditory structures (Winer et al., 1995). The major result is that strychnine application eliminated temporally sensitive facilitation. This suggests that glycinergic inhibition plays a prominent role in facilitated combination-sensitive responses and that these responses are created in the IC.

\section{MATERIALS AND METHODS}

Single-unit recordings were obtained from the IC in awake greater mustached bats (Pteronotus parnellii rubiginosus). Eleven bats captured in Trinidad and Tobago provided data for this report. Methods related to surgery, acoustic stimulation, and recording were detailed in a previous report (Portfors and Wenstrup, 1999a) and are summarized here. Procedures on the bats were approved by the Institutional Animal Care and Use Committee.

Surgical procedures. For surgery, bats were anesthetized with methoxyflurane (Metofane; Schering-Plough Animal Health, Omaha, NE) in combination with sodium pentobarbital (5 mg/kg, i.p.; Nembutal; Abbott Laboratories, North Chicago, IL) and acepromazine $(2 \mathrm{mg} / \mathrm{kg}$, i.p.; Med-Tech, Buffalo, NY). After the skull was exposed, a tungsten ground electrode was cemented into the skull over the right cerebral cortex. A metal pin was glued to the skull to position the head in the stereotaxic apparatus. After a topical antibiotic (tetracycline; American Cyanamid Company, Pearl River, NY) and local anesthetic (lidocaine; ElkinsSinns, Cherry Jill, NJ) were applied to the wound, the bat was returned to its holding cage to recover at least $1 \mathrm{~d}$ before recording.

On the first day of recording, bats were anesthetized with methoxyflurane to expose the IC ( $\sim 0.5 \mathrm{~mm}$ diameter hole). Recordings were obtained from awake animals held in a stereotaxic apparatus within a heated and humidified soundproof chamber. To reduce sound scatter, the chamber was lined with polyurethane foam, and the surface of the stereotaxic apparatus was covered with cotton. If a bat showed signs of discomfort, it was removed from the apparatus. Recording sessions generally lasted 4-6 hr.

Acoustic stimulation. Stimulus generation and data acquisition were computer-controlled. Two different tones were separately generated, switched, and attenuated. Each tone was 4 or $31 \mathrm{msec}$ total duration, including $0.5 \mathrm{msec}$ rise-fall times. Single-tone or two-tone stimuli were repeated four times per second. The signals were combined, amplified, then fed to a speaker $10 \mathrm{~cm}$ away from the bat and $25^{\circ}$ into the contralateral sound field. The speaker output was checked several times over the course of experiments with a calibrated microphone. There was a smooth, consistent decrease of $2.7 \mathrm{~dB}$ per $10 \mathrm{kHz}$ from 10 to $120 \mathrm{kHz}$. Fast Fourier transforms of the digitized microphone signal indicated that distortion components were not detectable $60 \mathrm{~dB}$ below the peak signal level.

Recording procedures. Single-unit recording and iontophoresis of drugs were performed using a micropipette electrode glued to a multibarreled pipette (Havey and Caspary, 1980). The recording electrode was filled with $0.9 \% \mathrm{NaCl}$ and had resistances of 15-25 $\mathrm{M} \Omega$. A five-barreled pipette (World Precision Instruments) was pulled and broken to a total tip diameter of $10-30 \mu \mathrm{m}$. The recording electrode was glued to the multibarreled pipette at an angle of $\sim 20^{\circ}$, protruding $10-25 \mu \mathrm{m}$ beyond it. Strychnine-HCl (10 mM, pH 3.5; Fluka, Milwaukee, WI) was placed in one or two barrels, and $0.9 \% \mathrm{NaCl}$ solution was placed in sum and control barrels. All solutions were prepared the day of recording. Each barrel was connected to one channel of a microiontophoresis current generator (model 6400; Dagan). The sum channel balanced currents in other channels being used to deliver or retain the drug.

Electrodes were positioned to record in the high-frequency representations of the IC (57-100 kHz) using surface landmarks and advanced with a hydraulic micropositioner. Extracellular action potentials from the recording electrode were amplified, then sent through a bandpass filter $(500-6000 \mathrm{~Hz})$ and a window discriminator. The output was then digitized at $10 \mathrm{kHz}$ and sent to the computer. Laboratory software generated peristimulus time histograms, raster displays, and statistics on neural responses based on 32 stimulus repetitions.

For all units the best frequency (the frequency requiring the lowest intensity to elicit stimulus-locked spikes) and threshold at best frequency (the lowest intensity that produce a consistent spike response) were first found, using single-tone burst stimuli. Then, using a two-tone stimulus paradigm, single units were tested for sensitivity to the combination of tones in the frequency range of the fundamental sonar call $(22-30 \mathrm{kHz})$ and at the best frequency (BF). BFs were in bands associated with higher harmonics of the sonar call. The best frequency signal was usually set 10 $\mathrm{dB}$ above threshold, then the frequency, intensity, and timing of the lower frequency signal were adjusted to obtain a strong combinationsensitive interaction. Under these conditions, combination sensitivity was evaluated quantitatively. Neurons were considered to show combinationsensitive facilitation if the response to the combination of the two signals from distinct frequency bands was at least $20 \%$ more than the sum of responses to the signals presented separately. The degree of facilitation was quantified as the index of interaction $(I)$, where $I=(\mathrm{Rc}-\mathrm{R} 1-$ $\mathrm{Rh}) /(\mathrm{Rc}+\mathrm{R} 1+\mathrm{Rh}) . \mathrm{Rc}, \mathrm{R} 1$, and $\mathrm{Rh}$ are, respectively, the responses of the neuron to the combination of the high- and low-frequency signals, low-frequency signal alone, and high-frequency signal alone. An interaction index of +1 indicates the strongest possible facilitation, and an index value of 0.09 indicates a $20 \%$ facilitation, corresponding to our definition of a facilitated combination-sensitive response.

In addition to stimulus-evoked responses to single and combination tones, we also collected data in no-stimulus conditions to assess the change in background discharge rate with strychnine. Whereas background discharge did not change significantly across the sample of units after strychnine application (mean difference, $1.44 \mathrm{spikes} / \mathrm{sec}$; paired $t$ test; $p>0.05$ ), in a few units the change was large, up to 14 spikes/sec. To minimize the influence of background discharge on the interaction index, spikes were usually counted in 50 or $60 \mathrm{msec}$ windows, sufficiently long for the responses to the 4 or $31 \mathrm{msec}$ signals used. In four units, there was still enough background discharge to bias the measurement of facilitation. In these units, the background discharge recorded in predrug and strychnine conditions was subtracted from the responses to the highfrequency, low-frequency, and combination stimuli.

If a combination-sensitive response was elicited, we examined quantitatively the sensitivity of the response to the timing, or delay, between the two signals. In most tests, delays were changed in 2 msec steps. For each delay test, we determined best delay (the delay between the highand low-frequency signal that elicits the strongest facilitated response). Data were collected before, during, and sometimes after application of strychnine. In the strychnine condition, the drug was iontophoresed for 8-12 min (+20-40 nA; retention current, $-15 \mathrm{nA})$ before testing began. Resistance for each barrel was constantly monitored; if the barrel became blocked (infinite resistance), the test was stopped.

\section{RESULTS}

This study describes effects of local strychnine application on responses of 24 single units from the mustached bat's IC. All units selected for testing showed temporally sensitive facilitation in response to presentation of sounds in two distinct frequency bands. The lower facilitating frequency was always in the 22-30 $\mathrm{kHz}$ band, the range associated with the bat's fundamental sonar signal. The higher facilitating frequency was in the range of 57-95 $\mathrm{kHz}$, with most in either the $58-60 \mathrm{kHz}(n=8)$ or $69-87 \mathrm{kHz}$ 

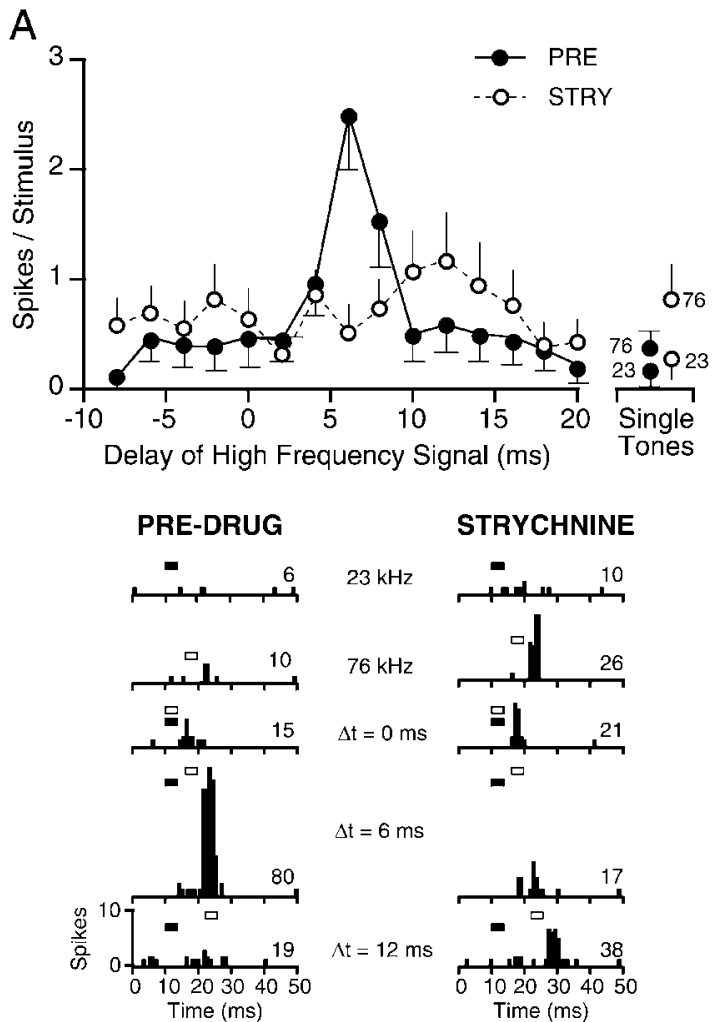
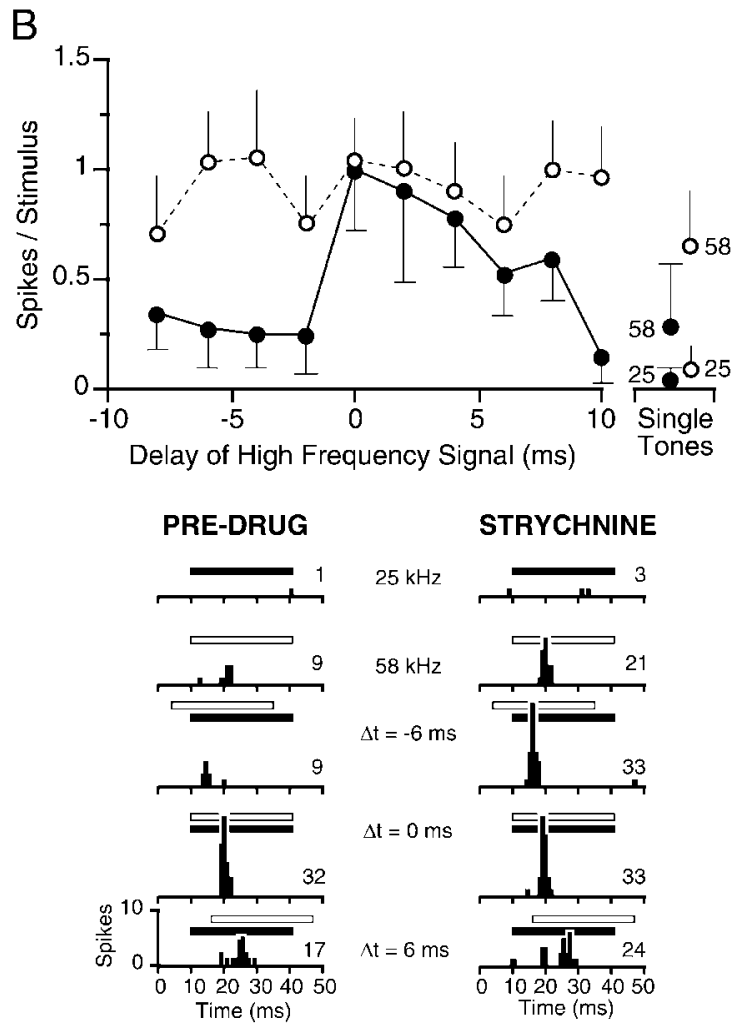

Figure 1. Strychnine eliminates facilitation in different types of combination-sensitive neurons in the mustached bat's IC. $A$, Effects of strychnine on an FM-FM single unit. Before application of the drug (PRE; filled dots and solid line), the unit showed strong facilitation when a $76 \mathrm{kHz}$ tone was presented 6 msec after a $23 \mathrm{kHz}$ tone. Magnitude of single-tone responses (numbers, tone frequency) shown to right of delay curve. Histograms show responses to 32 stimuli for single-tone responses and combinations at three delays (numbers at bottom right indicate total spikes). Above each histogram, horizontal bars show the timing and duration of low-frequency ( filled bars) or high-frequency (unfilled bars) tone bursts. With strychnine application (STRY; +39 nA, $10 \mathrm{~min}$ ), delay-tuned facilitation was eliminated. B, Effects of strychnine on a CF-related single unit. The unit showed a response facilitation when 25 and $58 \mathrm{kHz}$ tone bursts ( $31 \mathrm{msec}$ duration) were presented simultaneously $(0 \mathrm{msec}$ delay). Strychnine application ( $+32 \mathrm{nA}, 10 \mathrm{~min})$ eliminated facilitation; the unit responded only to the higher frequency signal. Error bars: $95 \%$ confidence limits.

( $n=13$ ) bands. These bands are associated with, respectively, the second harmonic of the constant frequency (CF) sonar component and the third harmonic of the frequency-modulated (FM) sonar component. All units were recorded from the tonotopic representation associated with their higher facilitation frequency, based on the frequency tuning of other neurons encountered in electrode penetrations.

As described elsewhere for IC neurons (Portfors and Wenstrup, 1999a), combination-sensitive neurons tuned to FM-related frequencies (called FM-FM neurons) show maximum facilitation when the higher frequency signal follows the lower frequency signal, with the best facilitating delay ranging from 1 to $20 \mathrm{msec}$ among neurons. Delay-tuned facilitation in an FM-FM neuron is illustrated in Figure $1 \mathrm{~A}$, filled circles and solid line. The unit responded weakly when presented with single tones in either of two frequency ranges, one centered at $23 \mathrm{kHz}$ and the other at 76 $\mathrm{kHz}$. However, the unit responded very strongly when the higher frequency signal was presented $6 \mathrm{msec}$ after the lower frequency signal. The response to the signal combination at best delay was $455 \%$ of the sum of the responses to the individual signals, corresponding to an interaction index value of 0.64 . Delay sensitivity was different for neurons tuned to $\mathrm{CF}$-related frequencies, as described elsewhere (Portfors and Wenstrup, 1999a). Among those in our sample, all but one were best facilitated when signals are presented simultaneously, i.e., at a delay of $0 \mathrm{msec}$ (Fig. $1 B$, filled circles and solid line).

Despite differences in delay sensitivity between FM-FM units and CF-related units, their facilitated responses were similarly affected by strychnine application. The major result, that strychnine eliminated delay-tuned facilitation, is illustrated by delay sensitivity curves and associated peristimulus time histograms in Figure 1. For the FM-FM unit in Figure $1 A$, iontophoretic application of strychnine eliminated the facilitation peak centered at $6 \mathrm{msec}$. Moreover, there was no facilitation at any delay (see criteria in Materials and Methods). For the CF-related unit in Figure $1 B$, strychnine application eliminated the delay selectivity of the response. Similar response magnitudes were obtained at all delays tested, corresponding to the response to the highfrequency stimulus presented alone. The application of strychnine eliminated delay-tuned facilitation in 22 of $24(92 \%)$ combination-sensitive units and substantially reduced the facilitation in the other two units (Fig. $2 A$ ). The decrease in facilitation across the sample was highly significant ( $p<0.001$, paired $t$ test). These results suggest that glycinergic inhibition is essential for the expression of delay-tuned facilitation in the mustached bat's IC.

Whereas strychnine application eliminated delay-tuned facilitation, its effect on the magnitude of the facilitated response to combination stimuli was not uniform (Fig. $2 B$ ). With strychnine, the response magnitude at the best delay decreased by $\geq 20 \%$ in 10 units (Fig. $1 A$ ). The response magnitude increased by $\geq 20 \%$ in seven units. For these units, facilitation was eliminated (six units) or reduced (one unit) with strychnine because the high-frequency response (and thus the response at nonoptimal delays) increased more than the combination response at best delay. The combina- 

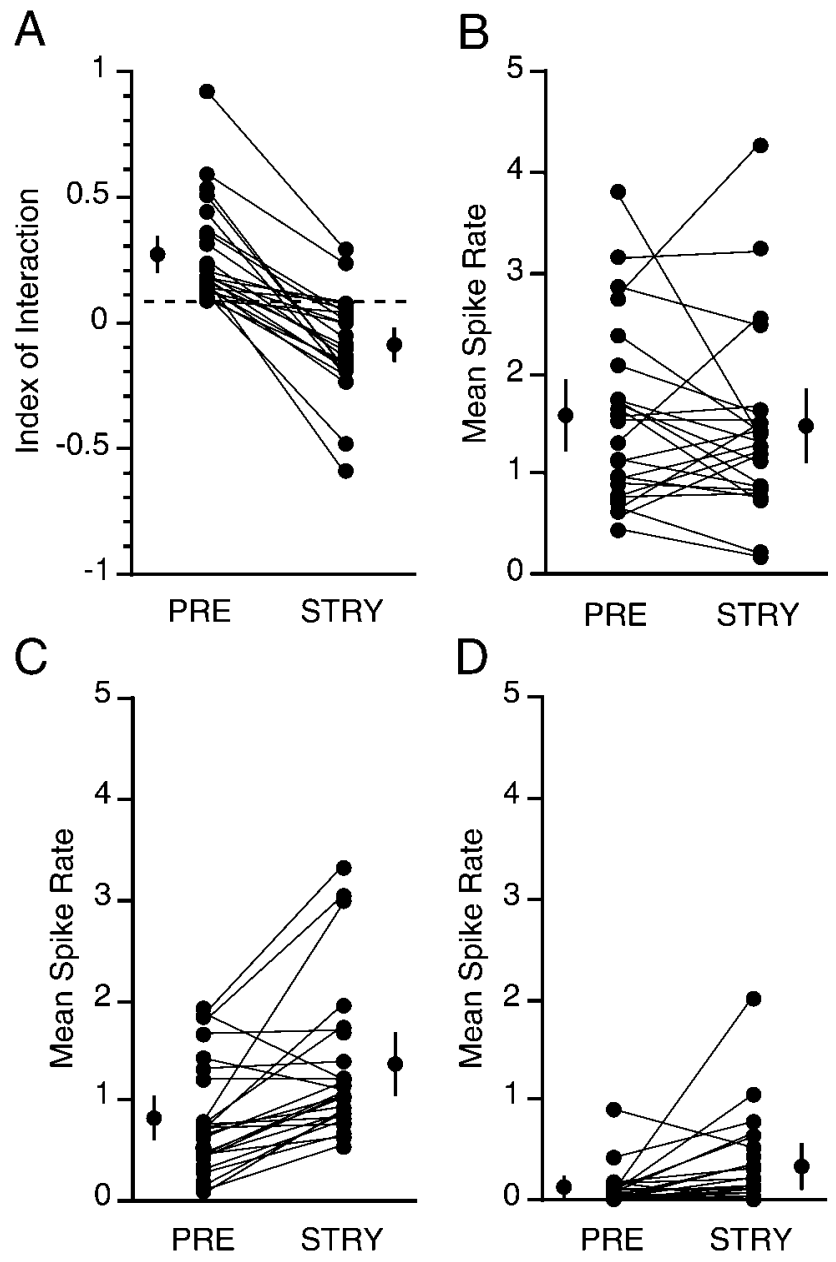

Figure 2. Strychnine eliminated or diminished facilitation in all neurons tested. $A$, Strength of facilitatory interaction for 24 combination-sensitive single units before and after strychnine application. Strength of interaction defined in the Materials and Methods. Dashed line indicates the minimum index value required for facilitation $(0.09$, or $120 \%$ facilitation $)$. Separate dots and error bars: mean and 95\% confidence limits for the population. Index values of -0.11 to -1.0 indicate combination-sensitive inhibition, in which the response to one signal is suppressed by the other signal. When facilitation was eliminated by strychnine, responses of 11 of 24 units showed combination-sensitive inhibition (Fig. $1 A$ ). This suggests the presence of an additional inhibitory input that is normally masked by glycine-dependent facilitation. $B-D$, For the 24 units, changes in response magnitude to: combination of tones at best delay $(B)$, high-frequency tones $(C)$, and low-frequency tones $(D)$. There was no consistent change in response to tone combinations, but responses to the high-frequency tone were significantly increased. There was no significant change in the low-frequency "response", which usually represented background activity.

tion response changed by $<20 \%$ in the remaining seven units (Fig. $1 B$ ). There was no significant change across the sample of units $(p>0.5$; paired $t$ test), nor was there a significant correlation between the change in the response magnitude at best delay and the change in facilitation as measured by the interaction index $(r=0.26 ; \mathrm{df}=22 ; p>0.05)$.

In contrast to the effect of strychnine on the facilitated response, responses to the high-frequency signal consistently increased with strychnine (Figs. 1, 2C). An increase in response by $\geq 20 \%$ occurred in 17 of 24 units, with only two units showing a decrease in response by $\geq 20 \%$. Across the population, there was a significant increase in the high-frequency response (paired $t$ test; $p<0.001$ ). There was no significant correlation between the change in the high-frequency response and the change in strength of facilitation $(r=-0.11 ; p>0.1)$.
There were few stimulus-locked responses to the lower frequency signal, in agreement with previous work (Portfors and Wenstrup, 1999). In both predrug and strychnine tests, only two units showed a clear, stimulus-locked, low-frequency response, and three others discharged a few spikes that may have been stimulus-locked (Fig. 1 $A$ ). On average, the low-frequency "response" increased after strychnine by 0.2 spikes/stimulus (Fig. $2 D)$, but it was not statistically reliable ( $p>0.05$; paired $t$ test). Because in most units there was no stimulus-locked response to the low-frequency tone, the spikes reflected background discharge. This is supported by tests showing that low-frequency "response" rates for both predrug and strychnine tests were not significantly different from background discharge rates obtained under the same conditions (paired $t$ tests: $p>0.05$ for each comparison).

The above results indicate that the strongest and most consistent effect of strychnine was the elimination of the response facilitation at the best delay and that this effect was unrelated to effects of strychnine on single-tone responses to either the higher or lower frequency signals.

\section{DISCUSSION}

\section{Combination-sensitive facilitation originates in the IC}

In the mustached bat's IC, $\sim 50 \%$ of neurons tuned to frequency ranges of the higher harmonics in the bat's sonar call display temporally selective, combination-sensitive facilitation (Portfors and Wenstrup, 1999a). These responses fall into two major categories, called FM-FM and CF-related, based on their sensitivity to different components in sonar signals and to the temporal relationship of signal elements. In both types of facilitated combination-sensitive responses, strychnine application eliminated or severely reduced the facilitation in all sampled neurons. The ability of strychnine to eliminate interactions between different spectral inputs suggests that the mechanisms underlying spectral integration act in the mustached bat's IC. This conclusion is supported by physiological studies of neurons in the lateral lemniscal nuclei. These nuclei provide the largest projection by far to combination-sensitive recording sites in the IC (Wenstrup et al., 1999), but only $3 \%$ of neurons in the lateral lemniscal nuclei show combination-sensitive facilitation (Portfors and Wenstrup, 1999b).

\section{Combination-sensitive facilitation depends on glycinergic inhibition}

The results suggest that glycinergic input to the IC is required for the expression of combination-sensitive facilitation. Because there are no glycinergic neurons in the IC, the source of glycinergic inputs to combination-sensitive, facilitated neurons in the IC must be in the auditory brainstem (Winer et al., 1995). Several auditory nuclei-the cochlear nucleus, the superior olivary nuclei, or the lateral lemniscal nuclei-could furnish glycinergic input (Winer et al., 1995; Kemmer and Vater, 1997). However, if the glycinergic input that contributes to facilitation is tuned to the lower frequency signal, then the most likely sources are the anteroventral cochlear nucleus and the ventral and intermediate nuclei of the lateral lemniscus. These brainstem nuclei are the only nuclei that satisfy two criteria: (1) they appear to contain labeling in their low-frequency regions after retrograde tracer deposits at FM-FM recording sites in the IC (Wenstrup et al., 1999), and (2) they contain glycine-immunopositive cell bodies (Winer et al., 1995; Kemmer and Vater, 1997). The critical role of glycine in combination-sensitive facilitation appears to rule out 
descending inputs as a source of the strychnine-sensitive facilitation, because there are no glycinergic neurons in the auditory forebrain (Winer et al., 1995).

In other studies of the IC, strychnine application reveals that glycinergic input has a range of inhibitory effects on response magnitude, temporal discharge pattern, and binaural response, each effect varying among a population of IC neurons (Faingold et al., 1991; Vater et al., 1992; Klug et al., 1995; Le Beau et al., 1996). Some of those inhibitory effects were also observed in this study, particularly the effect on the magnitude of responses to the best excitatory (i.e., high) frequency. In contrast, the main result of the present study is that glycinergic input contributes to response facilitation for nearly all combination-sensitive neurons.

The mechanism or mechanisms by which glycinergic input contributes to the facilitation are not understood. We hypothesize that a glycinergic input activates a postinhibitory rebound excitation. If that excitation coincides with excitation from another input, the response will be facilitated. Application of a glycine receptor antagonist should block the rebound excitation, and therefore the facilitation. Facilitatory responses to the combination of stimuli were consistently eliminated in the present study with strychnine. Glycine-evoked postinhibitory rebound has been observed or inferred in several studies. Casseday et al. (1994), studying auditory neurons selective for stimulus duration, found that strychnine application (and also bicuculline application) reduced or eliminated duration selectivity in a manner suggesting that glycine (and GABA) evokes a postinhibitory facilitation. Kotak and Sanes (1997), recording intracellularly from lateral superior olive neurons, observed a hyperpolarization-evoked rebound excitation that was strychnine-sensitive. Bertrand and Cazalets (1998) observed similar effects in spinal motoneurons. These results establish postinhibitory rebound as a plausible mechanism underlying the temporally sensitive facilitation of at least some combination-sensitive neurons.

There are other plausible mechanisms by which glycinergic inhibition contributes to a facilitated response, mechanisms in which glycinergic input acts as inhibition. These mechanisms are similar to those thought to underlie some binaural facilitation in the IC (Park and Pollak, 1994). For example, glycinergic input could suppress excitatory responses at nonoptimal delays between the low- and high-frequency tones, and this inhibitory effect would be blocked at the best delay by some other input. In this scenario, application of strychnine would elevate the response at nonoptimal delays, as occurred for the unit in Figure $1 B$. However, this mechanism is unlikely to explain the facilitation shown by 10 units, in which the response at best delay decreased with strychnine application (Fig. $1 A$ ).

Another plausible mechanism involves glycinergic disinhibition of another inhibitory input. This mechanism requires that facilitation is created by the convergence of excitatory inputs, some tuned to the lower best facilitatory frequency and some to the higher facilitatory frequency. An inhibitory neuron, most likely GABAergic, synapses onto the delay-tuned neuron to suppress the facilitated response. Glycinergic inhibition, timed appropriately, shuts off the GABAergic inhibition to permit the facilitated response at the best delay. For this scenario, strychnine application would be expected to remove the glycinergic inhibition of the GABAergic neuron, thus resulting in a suppression of the facilitated response. This agrees with our results in 10 of the neurons studied. However, strychnine application should also suppress response to either the low- or high-frequency stimuli, because glycinergic inhibition is not available to suppress the GABAergic input. Because we did not observe this effect throughout our sample, it seems unlikely that this mechanism applies to all of neurons tested here. Further work is required to test these possible mechanisms.

One class of combination-sensitive IC neurons, the FM-FM neurons, are maximally facilitated when a neurally delayed low frequency-evoked excitation (presumably from the emitted sonar pulse) coincides with an acoustically delayed high frequencyevoked excitation (presumably from an echo) (Suga et al., 1990; Olsen and Suga 1991; Portfors and Wenstrup, 1999a). Because the low-frequency signal occurs earlier in time than the highfrequency signal, the low-frequency excitation must be delayed within the CNS (i.e., have a longer latency) to achieve the coincidence. Among FM-FM neurons in the IC, for example, latencies for the low-frequency response, when present, are always longer than latencies for the high-frequency response. Moreover, the difference in the latencies correlates strongly with the best delay of a neuron, as predicted by coincidence detection models (Portfors and Wenstrup, 1999a). The glycinergic postinhibitory rebound mechanism that we believe underlies facilitation might also determine an FM-FM neuron's best delay. This could occur if the duration of the inhibitory period varied from neuron to neuron, causing variation in the timing of the rebound excitation to the low-frequency sound. This mechanism is similar to what is thought to occur in duration-tuned neurons (Casseday et al., 1994).

\section{Possible mechanism for analyzing complex vocal signals in vertebrates}

This study has implications beyond the coding of information in sonar echoes. The link between neurons analyzing sonar echoes and those analyzing other complex sounds, i.e., social vocalizations, has been strengthened by recent findings in the mustached bat. Cortical FM-FM neurons, believed to analyze target distance information in sonar (O'Neill and Suga, 1982), are also selective for particular mustached bat social vocalizations (Ohlemiller et al., 1996; Esser et al., 1997). Moreover, the majority of IC neurons tuned to frequencies outside the sonar bands show facilitatory or inhibitory interactions between distinct frequency inputs (Leroy and Wenstrup, 2000). The combinatorial responses of these neurons are similar to neurons in sonar-related frequency bands of the IC, but their frequency selectivity suggests they may analyze certain social vocalizations. These studies suggest that combinatorial responses to both sonar and social vocalizations may depend on common neural interactions, perhaps involving glycinergic inputs operating at the IC.

Combination-sensitive responses like those analyzing mustached bat sonar echoes are selective for social vocalizations in a wide range of vertebrates (Fuzessery and Feng; 1983; Margoliash and Fortune, 1992; Rauschecker et al., 1995). Recent studies show that spectral integration similar to combination sensitivity occurs in both primary and secondary auditory cortex (Brosch et al., 1999; Sutter et al., 1999; Fritz, 2000; Kadia et al., 2000), and these response properties are generally thought to reflect integrative mechanisms acting in auditory cortex. However, because combinatorial response properties in other species share similarities with combinatorial responses in the mustached bat, they may also share similarities in the underlying mechanisms and sites of integration. 


\section{REFERENCES}

Bertrand S, Cazalets JR (1998) Postinhibitory rebound during locomotionlike activity in neonatal rat motorneurons in vitro. J Neurophysiol 79:342-351.

Brosch M, Schulz A, Scheich H (1999) Processing of sound sequences in macaque auditory cortex: response enhancement. J Neurophysiol 82:1542-1559.

Casseday JH, Erlich D, Covey DE (1994) Neural tuning for sound duration: role of inhibitory mechanisms in the inferior colliculus. Science 264:847-850.

Doupe AJ (1997) Song- and order-selective neurons in the songbird anterior forebrain and their emergence during vocal development. J Neurosci 17:1147-1167.

Esser KH, Condon CJ, Suga N, Kanwal JS (1997) Syntax processing by auditory cortical neurons in the FM-FM area of the mustached bat Pteronotus parnellii. Proc Natl Acad Sci USA 94:14019-14024.

Faingold CL, Boersma Andeson CA, Caspary DM (1991) Involvement of GABA in acoustically-evoked inhibition in inferior colliculus neurons. Hear Res 52:201-216.

Fuzessery ZM, Feng AS (1983) Mating call selectivity in the thalamus and midbrain of the leopard frog (Rana p. pipiens): single and multiunit analyses. J Comp Physiol [A] 150:333-344.

Fritz J (2000) Spectral integration in the medial belt area of the macaque auditory cortex. Assoc Res Otolaryngol Abstr 23:86.

Havey DC, Caspary DM (1980) A simple technique for constructing "piggy-back" multibarrel microelectrodes. Electroencephalogr Clin Neurophysiol 48:249-251.

Kadia S, Snider R, Wang X (2000) Influence of stimulus components placed outside classical receptive field reveals harmonic structure of the auditory system. Assoc Res Otolaryngol Abstr 23:14.

Kemmer M, Vater M (1997) The distribution of GABA and glycine immunostaining in the cochlear nucleus of the mustached bat (Pteronotus parnellii). Cell Tissue Res 287:487-506.

Klug A, Park TJ, Pollak GD (1995) Glycine and GABA influence binaural processing in the inferior colliculus of the mustache bat. J Neurophysiol 74:1701-1713.

Kotak VC, Sanes DH (1997) Deafferentation weakens excitatory synapses in the developing central auditory system. Eur J Neurosci 9:2340-2347.

Le Beau FE, Rees A, Malmierca MS (1996) Contribution of GABAand glycine-mediated inhibition to the monaural temporal response properties of neurons in the inferior colliculus. J Neurophysiol 75:902-919.

Leroy SA, Wenstrup JJ (2000) Spectral integration in the inferior colliculus of the mustached bat. J Neurosci 20:8533-8541.

Margoliash D, Fortune ES (1992) Temporal and harmonic combinationsensitive neurons in the zebra finch's HVc. J Neurosci 12:4309-4326.

Mittmann DH, Wenstrup JJ (1995) Combination-sensitive neurons in the inferior colliculus. Hear Res 90:185-191.

Park TJ, Pollak GD (1994) Azimuthal receptive fields are shaped by
GABAergic inhibition in the inferior colliculus of the mustache bat. J Neurophysiol 72:1080-1102.

Ohlemiller KK, Kanwal JS, Suga N (1996) Facilitative responses to species-specific calls in cortical FM-FM neurons of the mustached bat. NeuroReport 7:1749-1755.

Olsen JF (1992) High-order auditory filters. Curr Opin Neurobiol $2: 489-497$.

Olsen JF, Suga N (1991) Combination-sensitive neurons in the medial geniculate body of the mustached bat: encoding of target range information. J Neurophysiol 65:1275-1269.

O'Neill WE (1985) Responses to pure tones and linear FM components of the CF-FM biosonar signal by single units in the inferior colliculus of the mustached bat. J Comp Physiol 157:797-815.

O'Neill WE, Suga N (1982) Encoding of target range and its representation in the auditory cortex of the mustached bat. J Neurosci $2: 17-31$.

Portfors CV, Wenstrup JJ (1999a) Delay-tuned neurons in the inferior colliculus of the mustached bat: implications for target distance analyses. J Neurophysiol 82:1326-1338.

Portfors CV, Wenstrup JJ (1999b) Origin of combination-sensitive neurons in the mustached bat: evidence from the nuclei of the lateral lemniscus. Soc Neurosci Abstr 25:396.

Rauschecker JP (1998) Cortical processing of complex sounds. Curr Opin Neurobiol 8:516-521.

Rauschecker JP, Tian B, Hauser M (1995) Processing of complex sounds in the macaque nonprimary auditory cortex. Science 268:111-114.

Suga N, O'Neill WE, Kujirai K, Manabe T (1983) Specificity of combination-sensitive neurons for processing of complex biosonar signals in auditory cortex of the mustached bat. J Neurophysiol 49:1573-1626.

Suga N, Olsen JF, Butman JA (1990) Specialized subsystems for processing biologically important complex sounds: cross-correlation analysis for ranging in the bat's brain. Cold Spring Harbor Symp Quant Biol 55:585-597.

Sutter ML, Schreiner CE, McLean M, O'connor KN, Loftus WC (1991) Organization of inhibitory frequency receptive fields in cat primary auditory cortex. J Neurophysiol 82:2358-2371.

Vater M, Habbicht H, Kossl M, Grothe B (1992) The functional role of GABA and glycine in monaural and binaural processing in the inferior colliculus of horseshoe bats. J Comp Physiol [A] 171:541-553.

Wenstrup JJ (1999) Frequency organization and responses to complex sounds in the medial geniculate body of the mustached bat. J Neurophysiol 82:2528-2544.

Wenstrup JJ, Mittmann DH, Grose CD (1999) Inputs to combinationsensitive neurons of the inferior colliculus. J Comp Neurol 409:509-528.

Winer JA, Larue DT, Pollak GD (1995) GABA and glycine in the central auditory system of the mustached bat: structural substrates for inhibitory neuronal organization. J Comp Neurol 355:317-353.

Yan J, Suga N (1996) The midbrain creates and the thalamus sharpens echo-delay tuning for the cortical representation of target-distance information in the mustached bat. Hear Res 93:102-110. 